\title{
The BepiColombo Mission
}

\author{
Rita Schulz \\ Research and Scientific Support Department of ESA, ESTEC, Postbus \\ 299, 2200 AG Noordwijk, The Netherlands
}

Peter Falkner, Anthony Peacock, Christian Erd, Nicola Rando

Science Payloads and Advanced Concepts Office of ESA, ESTEC, Postbus 299, 2200 AG Noordwijk, The Netherlands

Stefan Kraft

cosine Research B.V., Niels Bohrweg 11, 2333 CA Leiden, The Netherlands

\begin{abstract}
.
BepiColombo is an interdisciplinary mission to the planet Mercury which will provide the detailed information necessary to understand Mercury and its magnetospheric environment. The mission is envisaged to consist of three spacecrafts, the Mercury Planetary Orbiter (MPO), the Mercury Magnetospheric Orbiter (MMO) and the Mercury Surface Element (MSE). The mission went through a re-assessment with the aim of optimizing resources and and advancing the scientific return. Various mission scenarios were investigated and new payload concepts were adopted. The newly defined mission will be presented focusing on the launch scenario and the MPO reference payload.
\end{abstract}

\section{Introduction}

Mercury bears information, which unraveled might be the key to understanding the origin and early evolution of our solar system. The necessary details of its properties can, however, only be provided by a space mission. Nevertheless, only one spacecraft ever visited Mercury almost 30 years ago, when Mariner 10 performed three fly-byes in 1974-1975. Hence, precise characterization is still imminent. Drawing conclusions on the formation and evolution of Mercury requires a complete description of the planet and its environment. BepiColombo therefore is an interdisciplinary mission dedicated to a comprehensive investigation of the four basic components of the Mercury system: the planet's interior, surface, exosphere and magnetosphere. As such it will open a new frontier in the study of our solar system. The mission went through a re-assessment process to optimize resources and advance the scientific return. The new mission scenario is presented in a concise overview. 


\section{Mission Overview}

BepiColombo has been defined as a collaboration between ESA and ISAS/JAXA. It shall consist of three scientific elements, the Mercury Planetary Orbiter (MPO), the Mercury Magnetospheric Orbiter (MMO) and the Mercury Surface Element (MSE). The MMO, provided by ISAS/JAXA, focuses on investigating the wave and particle environment of the planet from an eccentric orbit. The spinning spacecraft accommodates mostly field, wave and particle instruments (ESA, 2000). The MPO and MSE are dedicated to the characterization of Mercury itself. The MPO is three-axis-stabilized and nadir pointing. Its low-eccentricity polar orbit will provide excellent spatial resolution over the entire planet surface. The MSE shall perform in-situ investigations of Mercury's surface and subsurface. For more details on the MSE see Thomas et al. (this volume).

During the re-assessment various mission profiles as well as new payload concepts and technologies were investigated in parallel. In the newly defined BepiColombo mission the MPO and MMO will no longer be launched separately on two Soyuz Fregats, but together on a single stack whereby the MPO will carry the MMO. This new scenario leaves the second Soyuz Fregat free for the launch of the MSE. The launch of the MPO/MMO complement is planned for 2012. Solar electric propulsion will be used for the journey to Mercury and chemical propulsion for the insertion of the spacecrafts into their dedicated orbits.

Accommodating both orbiters on a single Soyuz Fregat required to decrease the mass of the cruise composite, hence to optimize the mission in terms of hardware (scientific elements, propulsion modules) and mission analysis for cruise and orbit insertion (to save propellant mass). This has been achieved without compromising on the overall scientific return of the mission.

\subsection{The MPO Reference Payload}

A new payload concept was adopted which is based on a high level of integration. The MPO reference payload does no longer consist of individual instruments, but of the front ends of these instruments which share common subsystems such as DPU, electronics and power. In addition advantage was taken of the fact that new technologies and miniaturization techniques have been developed in recent years which allow alternative approaches in instrument design and increased instrument performances. The new concept led to a reduction of the payload mass by about $20 \mathrm{~kg}$ while at the same time more instruments could be accommodated. The MPO reference payload now also contains a thermal IR mapping spectrometer and radiometer, a neutral and ion particle analyzer, a limb pointing camera and a magnetometer. Figure 1 outlines the MPO reference payload and the topics addressed by the measurements.

Major effort was also put into optimizing the scientific return by defining the payload complement such that individual measurements can be interrelated and complement each other. The spatial resolution of the instruments measuring the elemental and mineralogical surface composition has for instance been adjusted to that of the camera, mapping the global surface morphology. This not only allows the morphological characterization of individual surface features (craters, basins, etc.) but also identification of compositional variations between features. The neutron spectrometer and the radiometer will take complemen- 


\section{MPO Reference Payload}

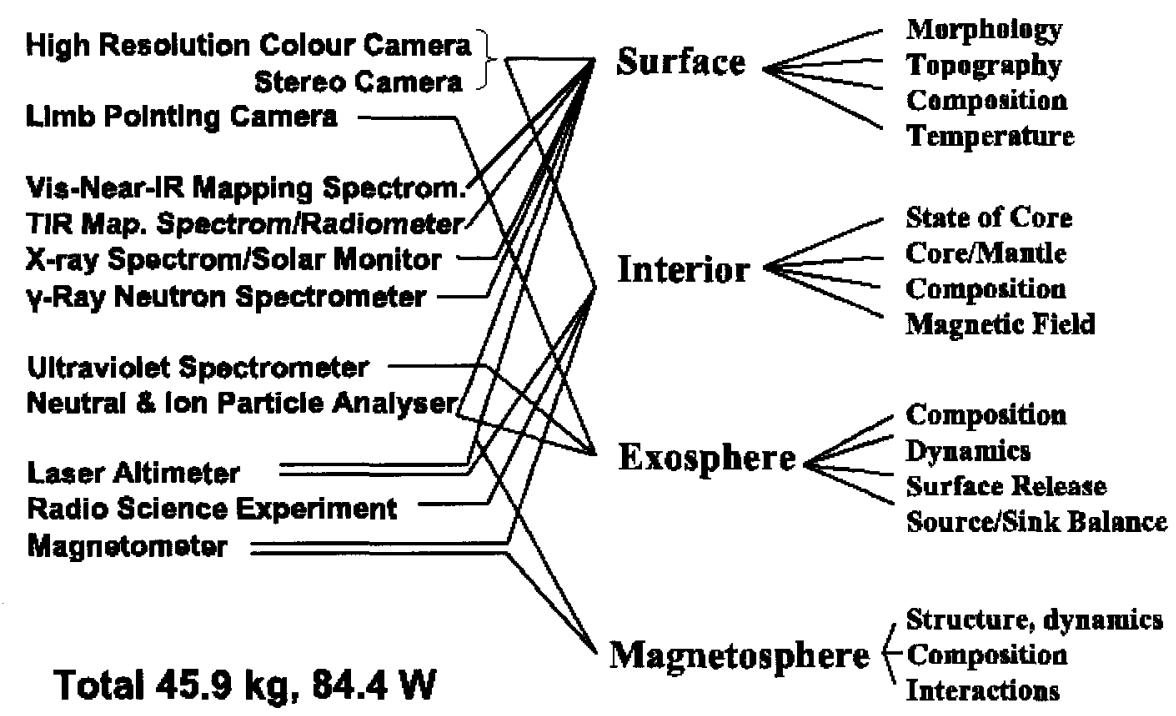

Figure 1. The MPO Reference Payload

tary measurements of the radar-bright spots in the polar regions to identify their composition. The measurements of the radio science experiment in combination with the high resolution camera and the laser altimeter will provide insight into the interior of the planet. Simultaneous measurements from MPO and MMO will resolve spatial and temporal ambiguities in the exosphere and magnetosphere that would arise from single point observations. For instance, having magnetometers on board both orbiters will allow to disentangle the contribution of the planetary magnetic field from that of the magnetosphere.

\section{Summary}

The MPO and MMO are on dedicated orbits optimized for the study of Mercury and its magnetosphere. Together they will provide the information needed to understand the planet and its environment, such as high accuracy measurements of the planet's interior structure, a full coverage of the planet surface at a resolution of $500 \mathrm{~m}$, the detailed structure of the planetary magnetic field and a complete characterization of Mercury's exosphere. Surface morphology will be correlated to surface composition. The MPO orbit provides optimal coverage of the polar regions. Hence the material of the radar-bright spots observed from 
ground and suspected to be either water ice or sulfur will be identified. A detection of sulfur would strongly support the presence of an at least partially molten core.

\section{Reference List}

\section{References}

ESA (2000) BepiColombo: An interdisciplinary cornerstone mission to the planet Mercury, System Technology Study Report, April 2000, ESA-SCI(2000)1.

Thomas, N., Schulz, R., \& Falkner, P. 2003, This volume, JD2 